\title{
Taste Sensor Chip for Portable Taste Sensor System
}

\author{
Shinichi Etoh*, Lingyan Feng ${ }^{1}$, Kenichi Nakashi², Kenshi Hayashi ${ }^{3}$, \\ Akira Ishii ${ }^{4}$ and Kiyoshi Toko ${ }^{3}$ \\ User Science Institute, Kyushu University, Fukuoka 819-0395, Japan \\ ${ }^{1}$ Graduate School of System Life Sciences, Kyushu University, Fukuoka 819-0395, Japan \\ ${ }^{2}$ Department of Electrical, Electronic and Computer Engineering, \\ Kyushu Institute of Technology, Fukuoka 804-8550, Japan \\ ${ }^{3}$ Gruduate School of Information Science and Electrical Engineering, \\ Kyushu University, Fukuoka 819-0395, Japan \\ ${ }^{4}$ Faculty of Design, Kyushu University, Fukuoka 815-8540, Japan
}

(Received January 21, 2008; accepted May 8, 2008)

Key words: taste sensor, lipid/polymer membranes, microfabrication, reference electrode, portable taste sensor

A taste sensor chip miniaturized on the basis of the one-chip-size receptor part of a conventional taste sensor, which can measure taste using lipid/polymer membranes, was fabricated. The reference electrode in the taste sensor chip realized the miniaturization and stabilization of potential using a multilayer structure of a pHEMA layer and other polymer layers. In this study, we improved the stability of the taste sensor chip during the measurement by changing the material of the partition of the taste sensor chip to plastic. We successfully integrated the working and reference electrodes on the chip. The portable taste sensor system was composed of the taste sensor chip, an integrated electric signal processing part and a laptop computer. We considered that the design of the portable taste sensor facilitates the easy manual measurement of taste. By realizing the taste sensor chip and the portable taste sensor, taste measurement can be easily performed anywhere.

\section{Introduction}

A taste sensor was invented by Toko and co-workers in Kyushu University. ${ }^{(1-3)}$ This sensor enables the analysis of the taste of foodstuffs, because the changes in the electric potential difference produced by lipid polymer membranes are measured. In other words, it is an instrument that enables the digitization of taste. The SA402B taste sensing system with some taste sensors has been produced by Intelligent Sensor Technology, Inc., and has been used for examining taste and developing new taste sensors in the food industry.

*Corresponding author: e-mail: etoh@usi.kyushu-u.ac.jp 
However, this instrument is the size of a desktop computer and is very heavy; therefore, it is difficult to carry. When a food sample is to be measured, it has to be carried to a laboratory or a facility with SA402B. The following advantages will be obtained if this instrument can be miniaturized: reductions in (1) the number of samples, (2) power consumption and (3) body weight. ${ }^{(4-9)}$ Thereby, the miniaturization of the taste sensor must be realized to measure taste anytime and anywhere.

Recently, the miniaturization of various sensors has been performed.(10-13) Also, research on miniaturization has been similarly performed for a potentiometeric sensor and various small reference electrodes. ${ }^{(14,15)}$ Moreover, research on sensor-arraying has been performed because it has become possible to integrate sensors.

We have conducted research aiming at the miniaturization of a taste sensor, and a taste sensor chip was developed. ${ }^{(16)}$ The taste sensor chip was miniaturized and integrated as the sensor probe part of a conventional taste sensing system. The integration of a reference electrode and a work electrode was performed on a glass chip as a substrate, which made it possible to measure an electric potential difference, and a fingertip-sized taste sensor was successfully fabricated. The reference electrode of the taste sensor chip had a multilayer structure. A poly-hydroxyethyl methacrylate (pHEMA) layer was deposited on the surface of a metal electrode layer, while successfully keeping the electric potential of the surface of a metal electrode constant by maintaining a high concentration of $\mathrm{KCl}$ solution in the layer. Stacking a polyvinyl chloride (PVC) layer and a filter layer on the pHEMA layer prevented the $\mathrm{KCl}$ from flowing out of the pHEMA layer. The work electrode was formed by directly depositing a blended lipid/polymer solution on a metal electrode surface. Furthermore, a multichannel taste sensor chip for several types of taste can be realized by integrating several types of work electrode.

In previous research, ${ }^{(16)}$ the stability of the electric potential of the taste sensor chip was adversely affected by the detachment of the lipid/polymer layer during the measurement. This problem was caused by the low adhesive strength between the PVC supporting the lipid/polymer layer and the glass of the substrate and partition.

In this paper, we propose an improved taste sensor chip in which the partition material is plastic. The adhesive strength of the plastic with PVC when tetrahydrofuran (THF) is the solvent is large, and it is expected that the stability of the taste sensor chip will be improved during measurement. The response of the electric potential of the taste sensor chip to a solution containing a taste substance is reported. Moreover, the electronic circuit that processes the electric signal acquired with the taste sensor chip is integrated, and a taste sensor system that is stored in a casing designed for easy measurement is proposed. We call this a portable taste sensor. The portable taste sensor can be used with a notebook PC for taste measurement anytime and anywhere.

\section{Experimental}

\subsection{Chip design}

Figure 1 shows the taste sensor chip design. On a glass substrate, titanium and silver/ silver chloride metals were used as electrodes. Titanium patterns were adhesive with 


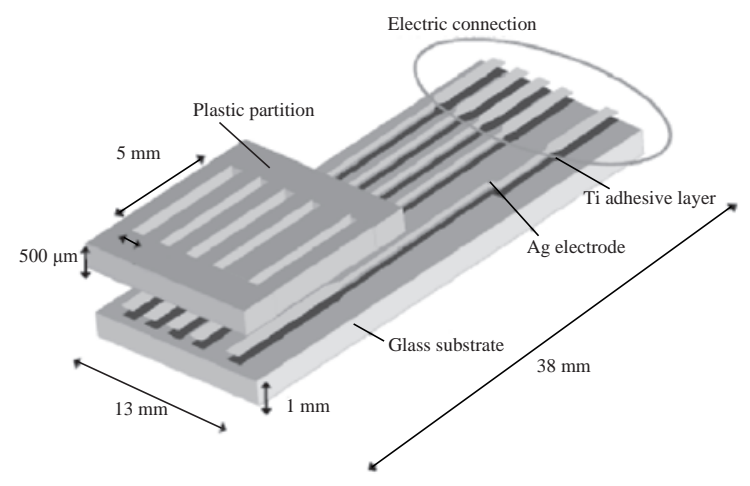

Fig. 1. Taste sensor chip design.

silver layers. To isolate each electrode of the sensor parts and to maintain the sufficient thickness of the lipid/polymer layers, a partition consisting of penetration cells of $5 \mathrm{~mm}$ $\times 1 \mathrm{~mm}$ in a plastic plate was formed. Silicone was used for adhesion between the glass substrate and plastic. Moreover, silicone was applied to protect the electrode surfaces from the sample solution.

\subsection{Chip fabrication}

Figure 2(a) shows the electrode substrate fabrication process. A glass plate was cut into $13 \mathrm{~mm} \times 38 \mathrm{~mm}$ pieces for use as glass substrates. A 500 - $\AA$-thick titanium layer was deposited by electron-beam evaporation on a glass substrate as an adhesive layer between the substrate and the silver layer. Next, on the titanium layer, a 2000- $\AA$-thick Ag layer was deposited by the same procedure. A photoresist was coated on the substrate by spin coating and then photolithography was performed to expose UV light through the photomask used to draw the electrode patterns. After development, the silver and titanium layers were etched by a mixed solution containing $\mathrm{NH}_{4} \mathrm{OH}$ and $\mathrm{H}_{2} \mathrm{O}_{2}$. The photoresist was removed from the substrate by washing in acetone and isopropanol (IPA). The silver/silver chloride layers were fabricated electrically by applying $0.5 \mathrm{~mA}$ for $10 \mathrm{~min}$. Figure 2(b) shows the fabrication of the plastic partition: first, a plastic PVC plate of 500 $\mu \mathrm{m}$ thickness was cut into $13 \mathrm{~mm} \times 13 \mathrm{~mm}$ square pieces. The hole of the sensor part was produced by drilling a plastic piece. The glass substrate with silver/silver chloride patterns was adhered to the partition made on a plastic piece using silicone as glue then was dried completely.

In this study, four lipid substances, palmitic acid, trioctyl methyl ammonium chloride (TOMA), tetra dodecyl ammonium bromide (TDAB) and phosphoric acid din-decyl ester (2C10), were used. Several lipids were mixed in each working electrode because the electric potential responses and specific qualitative response patterns were obtained for a specific taste substance. These lipid substances were added to the THF solution dissolved in PVC, and plasticizers of dioctyl phosphate (DOP), dioctyl phenyl 


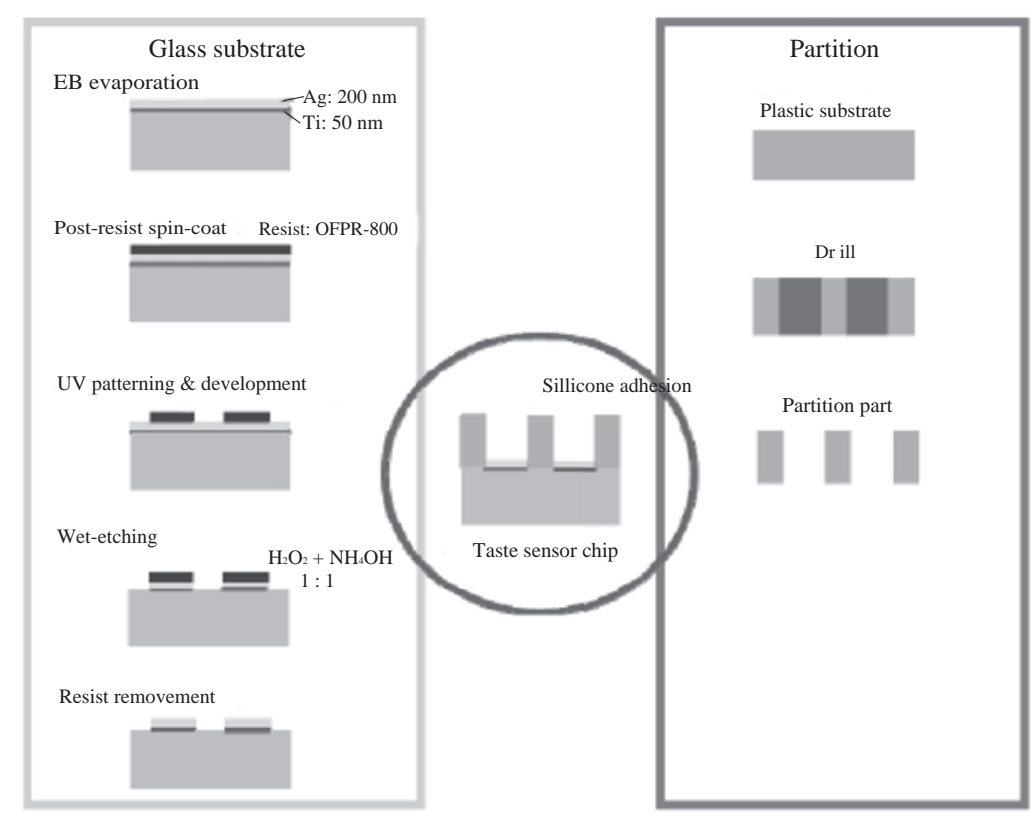

(a)

(b)

Fig. 2. Fabrication process for taste sensor chip.

phosphonate (DOPP) and $n$-tetradecyl alcohol (TDA) were also added. Then, each hybrid solution was mixed in a tube using a stirrer. Each lipid/polymer solution was poured into the fabricated cell on the taste sensor chip and dried completely. Working electrodes with sufficient layer thickness could be obtained for taste measurement by applying a lipid/polymer solution into the cell several times.

For the fabrication of the reference electrode, a HEMA monomer solution was prepared from a mixture of HEMA, ethylene glycol, demethoxy-2-phenylacetophenone and tetra ethylene glycol dimethacrylate. Then, this solution was added to glycerin and saturated $\mathrm{KCl}$ solution. The HEMA mixture was first injected into the reference electrode cell, and the polymerization to pHEMA was performed by UV irradiation of the cell for about 2 min. Next, after waiting for about 6 h to ensure that the polymerization to pHEMA was fully complete, a solution of mixed PVC and nitro phenyl octyl ether (NPOE) in THF solvent was applied to the pHEMA layer. This procedure was performed twice. Then, a solution for adjusting cellulose nitrate (CN) containing ethanol was stacked on the PVC/NPOE layer, which was used to prevent the elution of the $\mathrm{Cl}$ ions in the pHEMA layer.

\subsection{Measurement and chip operation}

In the measurement using the taste sensor chip, a mixed solution of $30 \mathrm{mM} \mathrm{KCl}$ and $0.3 \mathrm{mM}$ tartaric acid was used as the standard solution. Figure 3 shows the procedure of measurement using the taste sensor chip. The response potential $V$ was defined as 


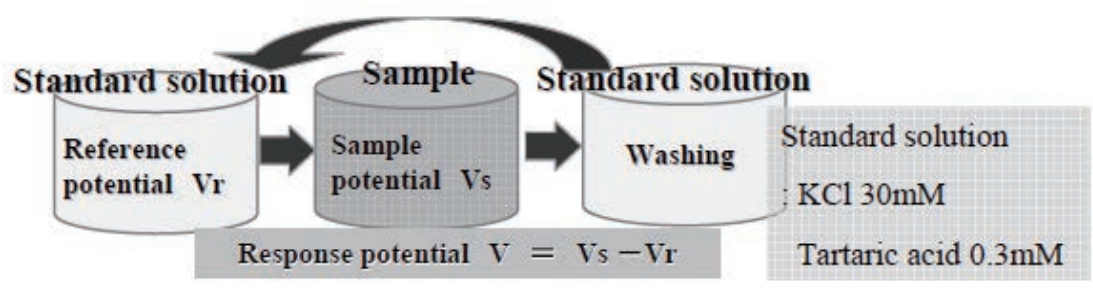

Fig. 3. Procedure of measurement using taste sensor chip.

the difference in electric potential between the standard and sample solutions containing taste substances. In this study, the electric potential was measured using agar reference electrodes, because the electric potential has yet to be measured accurately for the reference and working electrodes. First, the electric potentials of the taste sensor chip were stabilized in the standard solution, and then the reference electric potential $\left(V_{\mathrm{r}}\right)$ was measured. Next, the sample electric potentials $\left(V_{s}\right)$ were measured in sample solutions containing taste substances, and the sensor parts of the taste sensor chip were washed before each subsequent measurement was performed.

The electric potential signal from the taste sensor chip was amplified by an op-amp (OPA129P), inputted to the DAQ card and sent to a personal computer. The data was processed by LabVIEW 7.0 software, and the potential difference was recorded and expressed in real time.

\subsection{Chip structure}

Figure 4 shows a schematic cross-sectional view of the fabricated taste sensor chip. The working electrode is shown on the left-hand side, and the reference electrode is on the right-hand side. In previous research, the partition was fabricated from photosensitive glass, which had a low adhesive strength with PVC. As a result, the lipid/polymer layer was sometimes removed from the partition during the measurement. In this study, we changed the partition material to PVC. The working electrode was directly injected into the prepared lipid/polymer solution in the cell. The lipid/polymer layer adhered strongly to the PVC partition. Consequently, the stability of the working electrode was improved. To obtain sufficient layer thickness of the working electrode required for the potential measurement, the injection of the lipid/polymer solution was performed twice. The resulting layer thickness was about $100 \mu \mathrm{m}$.

The reference electrode had a multilayer structure consisting of a pHEMA layer, a PVC layer and a filter layer. As stated above, in the reference electrode, the PVC layer strongly adhered to the PVC partition. By using high-concentration $\mathrm{KCl}$ solution to wet the pHEMA layer, the electric potential of the $\mathrm{Ag} / \mathrm{AgCl}$ electrode surface was fixed. Furthermore, in order to prevent the outflow of the $\mathrm{KCl}$ solution in the pHEMA layer, the electric potential was fixed by stacking a PVC layer and a filter layer. By laminating the polymers on the $\mathrm{Ag} / \mathrm{AgCl}$ electrode, a new reference electrode, which was much smaller than the conventional reference electrode, was realized on the chip substrate. A disposable taste sensor chip can possibly be fabricated using this new structure. 


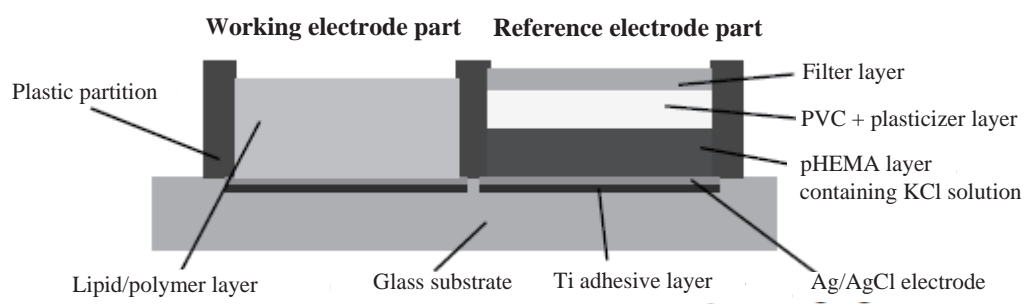

Fig. 4. Cross-sectional view of fabricated taste sensor chip.

\section{Results and Discussion}

Figure 5 shows the electric potential in the reference electrode of the taste sensor chip in $1 \mathrm{M} \mathrm{KCl}$ solution. In the measurement of the electric potential, the fabricated taste sensor chip with the reference electrode was immersed in $1 \mathrm{M} \mathrm{KCl}$ solution. In previous research, ${ }^{(16)}$ the reference electrode of the taste sensor chip was fabricated with a photosensitive glass partition and the stability of the electric potential was examined. Results indicate that the stability was maintained for $12 \mathrm{~h}$. From the start of measurement, an electric potential change of $1 \mathrm{mV}$ in $1 \mathrm{~h}$ appeared after $5 \mathrm{~h}$. The electric potential change became smaller after $5 \mathrm{~h}$, and an electric potential change of $0.2 \mathrm{mV}$ in $1 \mathrm{~h}$ was observed after $10 \mathrm{~h}$ of measurement. Subsequently, the electric potential remained stable for about $24 \mathrm{~h}$. As can be observed, the stability of the electric potential in the reference electrode of the taste sensor chip was improved by changing the partition material from glass to plastic.

Figure 6 shows the electric potential response in the working electrode of the taste sensor chip with a lipid/polymer layer (mixture of TOMA, DOP and DOPP) for different concentrations of five taste substances ( $\mathrm{KCl}$, tartaric acid, monosodium glutamate (MSG), quinine and tannin). The electric potential was increased by increasing the concentration of tartaric acid, and decreased by increasing the concentrations of MSG and tannin. However, the electric potential response was not clear for $\mathrm{KCl}$ and quinine. Also, compared with the conventional taste sensor SA402B, the electric potential response was smaller for MSG; however, its qualitative characteristic was similar.

Figure 7 shows the electric potential response in the working electrode using the taste sensor chip with a lipid/polymer layer (mixture of palmitic acid and DOPP) for different concentrations of the five taste substances. Compared with the previous result in Fig. 6, a much greater electric potential response was observed for changes in the concentration of quinine. As a result, by changing the lipid or plasticizer, the response to a taste substance was changed. The electric potential responses were similar to those of SA402B, particularly for tartaric acid.

Figure 8 shows the electric potential response in the working electrode using the taste sensor chip with a lipid/polymer layer (mixture of TDAB, DOPP and TDA) for different concentrations of the five taste substances. This working electrode only responded significantly to $\mathrm{KCl}$, and the electric potential response agreed with that of SA402B. 


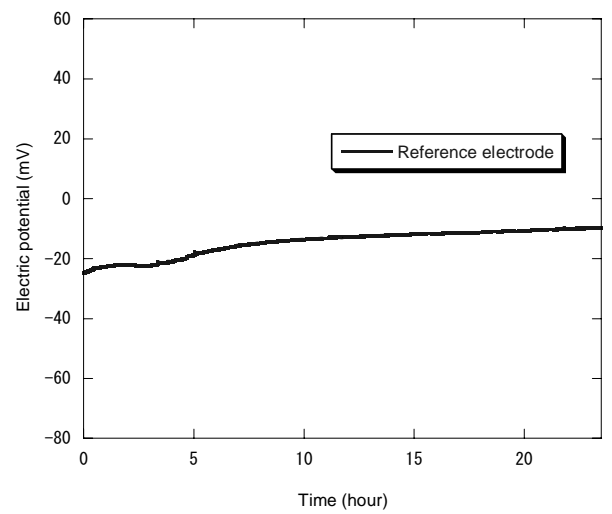

Fig. 5. Electric potential in reference electrode of taste sensor chip in $1 \mathrm{M} \mathrm{KCl}$ solution.

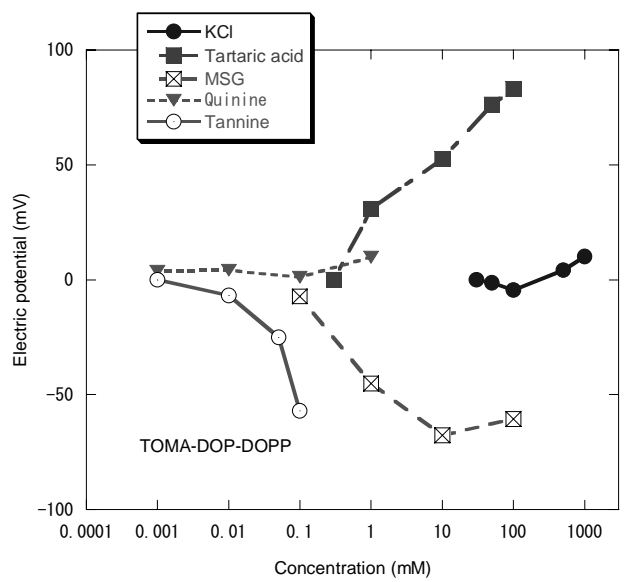

Fig. 6. Electric potential response in working electrode using taste sensor chip with lipid/polymer layer (mixture of TOMA, DOP and DOPP).

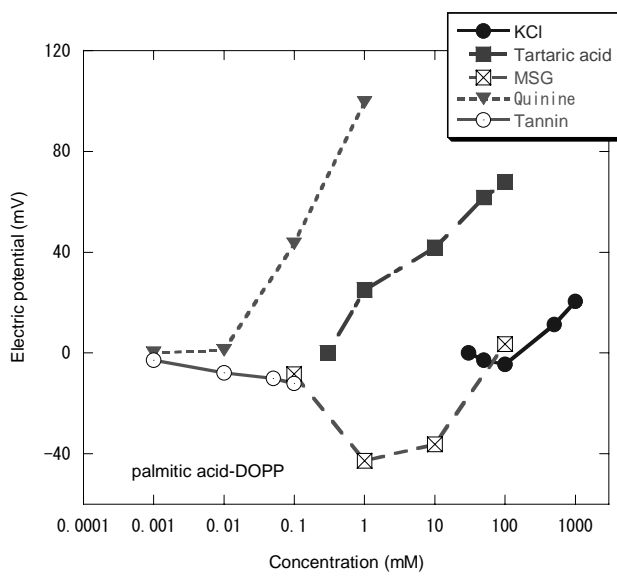

Fig. 7. Electric potential response in working electrode using taste sensor chip with lipid/polymer layer (mixture of palmitic acid and DOPP). 


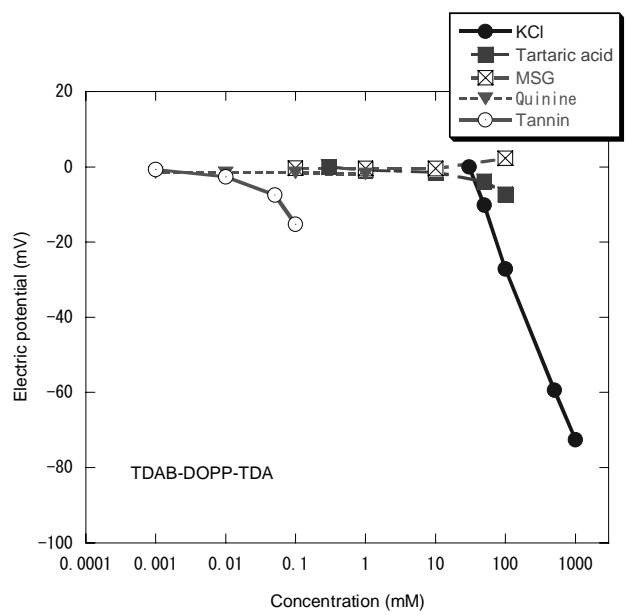

Fig. 8. Electric potential response in working electrode using taste sensor chip with lipid/polymer layer (mixture of TDAB, DOPP and TDA).

Figure 9 shows the electric potential response in the working electrode using the taste sensor chip with a lipid/polymer layer (mixture of TDAB and DOPP) for different concentrations of the five taste substances. Compared with the previous result in Fig. 8, a greater electric potential response was observed for $\mathrm{KCl}$ and tannin. However, the electric potential response was small for tannin compared with the conventional taste sensor SA402B. Therefore, the taste sensor chip measured the taste quality by combining the working electrodes.

We propose a prototype portable taste sensor fabricated by miniaturizing and integrating the amplification and signal-processing parts required for the taste measurement, as shown in Fig. 10. This sensor can easily be used to measure taste. This device in conjunction with a laptop computer, which operates the data treatment from the portable taste sensor, can easily measure the taste of food anywhere.

\section{Conclusions}

A taste sensor chip placed in the one-chip-size receptor part of a taste sensor was fabricated. The working and reference electrodes were integrated on a glass substrate. The reference electrode realized a reduction in electric potential using a multilayer structure of pHEMA and polymers. In this study, the material of the partition of the taste sensor chip was changed to plastic. As a result, the stability of the electric potential of the reference electrode was improved. The potential response of the working electrode and the stability of the electric potential of the reference electrode in the taste sensor chip were sufficient for taste measurement. A portable taste sensor was thus fabricated with a design that facilitated easy taste measurement. The portable taste sensor system was composed of the taste sensor chip, an integrated electric signal processing part and a laptop computer. By realizing the portable taste sensor system, taste measurement can now be easily performed anywhere. 


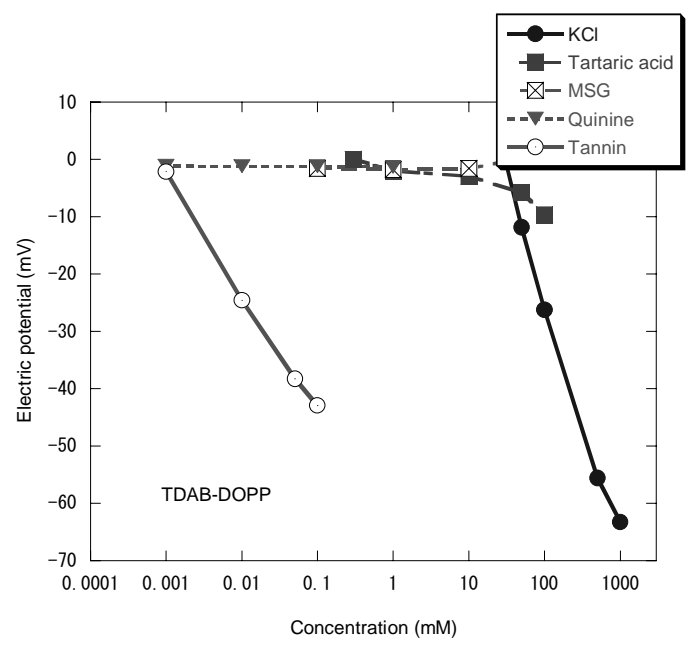

Fig. 9. Electric potential response in working electrode using taste sensor chip with lipid/polymer layer (mixture of TDAB and DOPP).

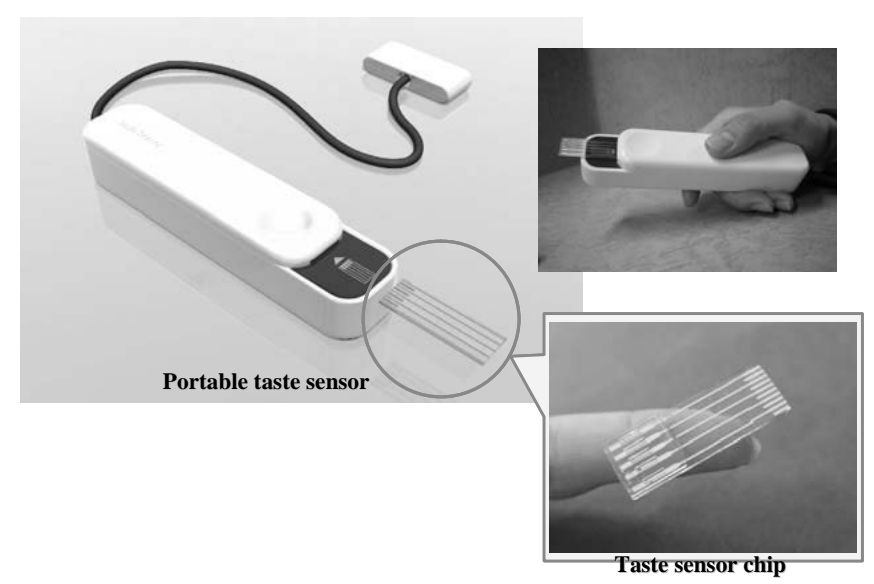

Fig. 10. Portable taste sensor with taste sensor chip.

\section{Acknowledgements}

This study was partially supported by the User Science Institute of Kyushu University.The User Science Institute of Kyushu University was established in 2004 as an across-the-board organization by the Ministry of Education, Culture, Sports, Science and Technology as one of its "Strategic Research Center Developing Programs" funded by its Special Coordination Funds for Promoting Science and Technology. 


\section{References}

1 K. Toko: Biomimetic Sensor Technology (Cambridge University Press, Cambridge, 2000).

2 K. Toko: Tech. Dig. Int. Conf. MEMS (2004) 201.

3 K. Hayashi, M. Yamanaka, K. Toko and K. Yamafuji: Sens. Actuators B 2 (1990) 205.

4 D. J. Harrison, A. Manz, Z. Fan, H. Lüdi and H. M. Widmer: Anal. Chem. 64 (1992) 1926.

5 H. Nakanishi, H. Abe, T. Nishimoto and A. Arai: Bunseki Kagaku 47 (1998) 361.

6 M. Vazquez, G. McKinley, L. Mitnik, S. Desmarais, P. Matsudaira and D. Ehrlich: Anal. Chem. 74 (2002) 1952.

7 S. C. Jacobson, R. Hergenröder, L. B. Koutny, R. J. Warmack and J. M. Ramsey: Anal. Chem. 66 (1994) 1107.

8 M. Tokeshi, T. Minagawa and T. Kitamori: Anal. Chem. 72 (2000) 1711.

9 S. Etoh, T. Fujimura, R. Hattori and Y. Kuroki: Jpn. J. Appl. Phys. 42 6B (2003) 4093.

10 A. Uhlig, E. Lindner, C. Teutloff, U. Schnakenberg and R. Hintsche: Anal. Chem. 69 (1997) 4032.

11 B. Lakard, O. Segut, S. Lakard, G. Herlem and T. Gharbi: Sens. Actuators B 122 (2007) 101.

12 L. Tymecki and R. Koncki: Sens. Actuators B 113 (2006) 782.

13 J. H. Shim, J. Kim, G. S. Cha, H. Nam, R. J. White, H. S. White and R. B. Brown: Anal. Chem. 79 (2007) 3568.

14 S. K. Kim, H. Lim, T. D. Chung and H. C. Kim: Sens. Actuators B 115 (2006) 212.

15 W. Y. Liao and T. C. Chou: Anal. Chem. 78 (2006) 4219.

16 S. Etoh, M. Iwakura, K. Nakashi, K. Hayashi and K. Toko: Proc. 2006 Int. Conf. Microtechnology in Medicine and Biology (2006) TA6. 